\title{
Manganese superoxide dismutase promotes anoikis resistance and tumor metastasis
}

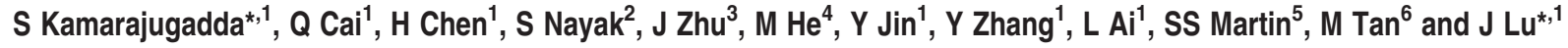

Normal cells require adhesion to extracellular matrix for survival. Cell detachment causes a drastic increase in reactive oxygen species (ROS) that promotes anoikis. In the present study, we observed that upon detachment from matrix, human mammary epithelial cells strongly upregulate manganese superoxide dismutase (MnSOD, or SOD2), a principal mitochondrial antioxidant enzyme that detoxifies ROS through dismutation of superoxide. Induction of MnSOD by cell detachment is dependent on the $\mathrm{NF} \kappa \mathrm{B}$ transcription factor. Detachment of mammary epithelial cells potently increases mitochondrial superoxide levels, which are further elevated by depletion of MnSOD in suspended cells. Consequently, cells depleted of MnSOD are hypersensitive to matrix detachment and exhibit increased anoikis. These results suggest that detachment-induced MnSOD counters mitochondrial superoxide accumulation and confers anoikis resistance. Taken together with our previous finding that detached cells evade excessive ROS production by attenuating oxidative metabolism of glucose, we conclude that mammary epithelial cells coordinate their responses to detachment through increasing MnSOD and decreasing ROS generation from mitochondrial glucose oxidation, thereby mitigating anoikis. Anoikis is a barrier to tumor metastasis. Indeed, MnSOD expression is elevated in human breast cancer metastases compared with primary tumors. Expression of MnSOD correlates with histologic tumor grades in human cancer and contributes to cancer cell's resistance to anoikis. Our study suggests that inhibition of ROS detoxification coupled with stimulation of glucose oxidative metabolism may be an efficient strategy to enhance anoikis and block metastasis. Cell Death and Disease (2013) 4, e504; doi:10.1038/cddis.2013.20; published online 21 February 2013

Subject Category: Cancer metabolism

In normal tissues, adhesion to extracellular matrix (ECM) components activates intracellular pro-survival signaling pathways. Disruption of this cell-matrix contact induces a type of apoptosis termed as anoikis. ${ }^{1}$ Mitochondria have a central role in the induction of anoikis. Mitochondrial intermembranous space (IMS) contains a number of key proapoptotic factors, such as cytochrome c, which trigger apoptosis if released into the cytosol. Cell detachment causes upregulation and translocation of several $\mathrm{BH} 3$-only proteins (e.g., BMF and BIM) to mitochondria, where they activate the pro-apoptotic BCL-2 family members BAK and BAX to stimulate mitochondrial outer-membrane permeabilization (MOMP) that facilitates the release of IMS apoptogenic factors. ${ }^{1,2}$ In addition, cells detached from matrix markedly increase the amount of reactive oxygen species (ROS) in mitochondria. ${ }^{3}$ While low levels of ROS activate various signaling pathways, excessive ROS can irreversibly damage cellular macromolecules and cause cell death (including apoptosis). ROS may activate intrinsic mitochondrial apoptotic pathway in part through lipid peroxidation. Cytochrome $\mathrm{c}$ is normally retained to the mitochondrial inner membrane due to its association with cardiolipin, a mitochondria-specific anionic phospholipid. ROS peroxidate cardiolipin, resulting in dissociation and liberation of cytochrome $c,{ }^{4}$ which may then freely diffuse through porous mitochondrial outer membrane (as a consequence of MOMP). Therefore, matrix detachment leads to efficient release of pro-apoptotic factors from the mitochondrial IMS to the cytosol, and consequently, activation of the caspase cascade and apoptosis.

Consistent with the pro-anoikis activity of ROS, treatment with a variety of antioxidants suppresses anoikis, ${ }^{3,5}$ supports anchorage-independent cell growth/survival in soft agar, and delays luminal clearance during acinar morphogenesis in vitro. ${ }^{6}$ However, the role of ROS in cell viability has been controversial. It was reported that when suspended cells attach to ECM, the adhesion process induces a transient burst of ROS, which in turn activate signaling pathways that inhibit apoptosis. ${ }^{7}$ It was thus suggested that ROS may instead confer pro-survival activity and resistance to anoikis. ${ }^{7}$ While the discrepancy is likely attributed to different cellular contexts used in these studies (e.g., detachment versus attachment), nevertheless, the cellular effect of oxidative stress depends

\footnotetext{
${ }^{1}$ Department of Biochemistry and Molecular Biology, Gainesville, FL, USA; ${ }^{2}$ Department of Pediatrics, University of Florida College of Medicine, Gainesville, FL, USA; ${ }^{3}$ Department of Pathology, The First Hospital of Lanzhou University, Lanzhou, China; ${ }^{4}$ Department of Surgery, Gansu Provincial Tumor Hospital, Lanzhou, China; ${ }^{5}$ Greenebaum $\mathrm{NCl}$ Cancer Center, University of Maryland School of Medicine, Baltimore, MD, USA and ${ }^{6}$ Mitchell Cancer Institute, University of South Alabama, Mobile, AL, USA

*Corresponding author: S Kamarajugadda or J Lu, Department of Biochemistry and Molecular Biology, University of Florida, PO Box 100245, Gainesville, FL 32610, USA. Tel: 352273 8200; Fax: 352273 8222; E-mail: susha80@ufl.edu or jrlu@ufl.edu

Keywords: anoikis; ROS; MnSOD; NF $\kappa$ B; metastasis; NRF2

Abbreviations: DCA, dichloroacetate; ECM, extracellular matrix; ER, estrogen receptor; ERR, estrogen-related receptor; HMECs, human mammary epithelial cells; IMS, inter-membranous space; MnSOD, manganese superoxide dismutase; MOMP, mitochondrial outer-membrane permeabilization; PDH, pyruvate dehydrogenase; PDK, pyruvate dehydrogenase kinase; PgR, progesterone receptor; ROS, reactive oxygen species; RT-PCR, reverse transcription-polymerase chain reaction; shRNA, short-hairpin RNA; TCA, tricarboxylic acid cycle

Received 08.8.12; revised 04.1.13; accepted 09.1.13; Edited by A Finazzi-Agró
} 
on the level of ROS and cell's capacity to respond to and cope with ROS.

Mitochondria are a major source of intracellular ROS. Under physiological conditions, ROS are constantly generated as by-products of aerobic metabolism in the mitochondria. ${ }^{8}$ During mitochondrial respiration, the tricarboxylic acid (TCA) cycle produces reducing equivalents that are the source for electrons. Electron transfer between mitochondrial electron transport complexes establishes a proton gradient for ATP synthesis. However, electrons can escape from the electron transport chain, and react with oxygen molecule to form superoxide anion, the first member in a plethora of ROS. Approximately $1-5 \%$ of the total oxygen consumed during normal respiration is converted to superoxide radicals. Superoxide is highly reactive and toxic. Superoxide can also react with nitric oxide, another free radical formed by mitochondrial nitric oxide synthase, to generate the highly reactive peroxynitrite. These ROS can inflict severe damage to mitochondrial lipids, proteins, and nucleic acids. To counter the deleterious effect of ROS, cells are equipped with antioxidant systems to detoxify ROS and prevent them from accumulating at high concentrations. ${ }^{9}$ The mitochondrialocated manganese superoxide dismutase (MnSOD, or SOD2) efficiently converts superoxide to the less reactive hydrogen peroxide that may break down further into water and dioxygen by other enzymatic and non-enzymatic antioxidants. ${ }^{9}$ In general, the balance between the production and elimination of ROS leads to homeostasis. Because superoxide primarily arises from mitochondria, mitochondrial MnSOD has a pivotal role in detoxification of ROS.

We and others recently discovered that following matrix detachment, untransformed human mammary epithelial cells robustly upregulate pyruvate dehydrogenase $(\mathrm{PDH})$ kinase 4 (PDK4) to inhibit PDH and attenuate the flux of glycolytic carbon to the TCA cycle. ${ }^{5,10}$ We further demonstrated that this metabolic response reduces mitochondrial respiration-associated generation of ROS. Depletion of PDK4 or forced activation of $\mathrm{PDH}$ augments oxygen consumption and ROS production in suspended cells, leading to increased anoikis. ${ }^{5}$ Therefore, detached cells reprogram glucose metabolism to evade excessive ROS production, thereby alleviating anoikis. The findings illustrate metabolic modulation of anoikis and reinforce the vital role of oxidative stress in anoikis.

Because MnSOD is the principal antioxidant enzyme that detoxifies mitochondrial ROS, we hereby examined its potential involvement in anoikis. We found when detached from matrix, human mammary epithelial cells strongly upregulate $\mathrm{MnSOD}$ via the $\mathrm{NF} \kappa \mathrm{B}$ transcription factor to reduce oxidative stress and acquire anoikis resistance. Depletion of MnSOD leads to increased accumulation of mitochondrial superoxide in suspended mammary epithelial cells, and enhances anoikis. Therefore, in response to matrix detachment, cells activate two parallel programs: (1) attenuation of oxidative metabolism of glucose to decrease mitochondrial ROS production ${ }^{5}$ and (2) enhancement of antioxidant capacity to detoxify ROS, which allow them to lower oxidative stress and prolong survival in suspension. Elevated expression of MnSOD is associated with advanced tumor grades and breast cancer metastasis, supporting its role in anoikis resistance and tumor progression.

\section{Results}

Induction of MnSOD by matrix detachment in mammary epithelial cells. Given the importance of mitochondrial ROS in anoikis and MnSOD in ROS detoxification, we decided to examine the potential significance of MnSOD in cells detached from matrix. We compared MnSOD expression in cells under attached and suspension culture conditions. Both primary human mammary epithelial cells (HMECs) and MCF10A mammary epithelial cell line remarkably upregulated MnSOD RNA levels upon detachment (Figure 1a). Induction of MnSOD was evident by 12 and $6 \mathrm{~h}$ after suspension of HMECs and MCF10A cells, respectively (Figures $1 \mathrm{~b}$ and $\mathrm{c}$ ). Based on this time course, MnSOD seems to be induced more rapidly than PDK4 following cell detachment. ${ }^{5}$ These observations demonstrate that human mammary epithelial cells increase expression of the MnSOD antioxidant enzyme in response to matrix detachment.

NF $\kappa \mathrm{B}$ is essential for induction of MnSOD upon cell detachment from ECM. The mechanism by which MnSOD was activated in suspended cells was unknown. Because we previously identified the estrogen-related receptor (ERR $\gamma)$ as the main activating factor for the strong induction of PDK4 by matrix detachment, ${ }^{5}$ we tested if induction of MnSOD was also dependent on ERR $\gamma$. In ERR $\gamma$-depleted MCF10A cells, MnSOD was still potently induced following cell detachment (Supplementary Figure S1A), which is in contrast to PDK4
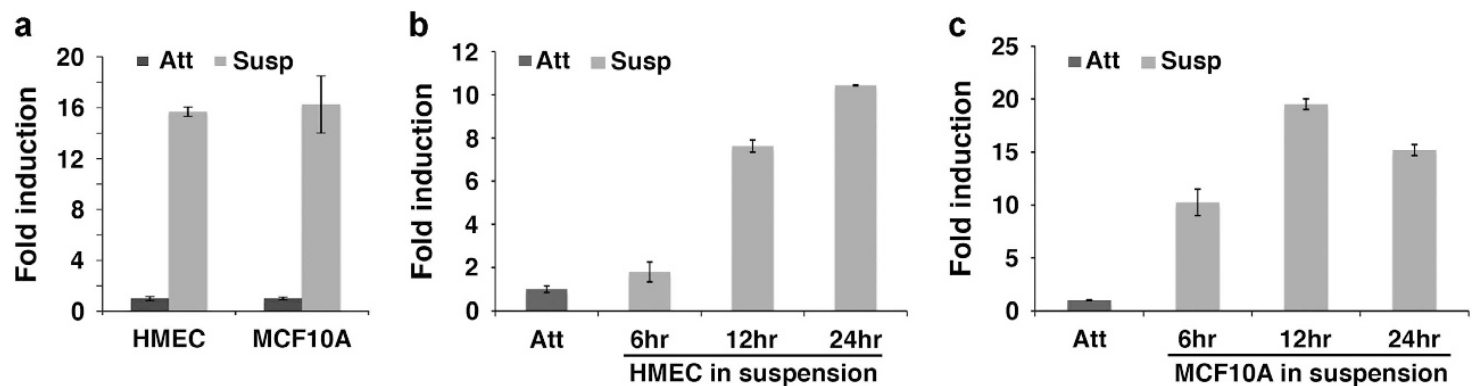

Figure 1 Matrix detachment induces MnSOD in mammary epithelial cells. (a) Quantitative RT-PCR for MnSOD in HMEC and MCF10A cells under attached (Att) and suspended (Susp) conditions after overnight incubation, (b) time course of MnSOD induction measured by quantitative RT-PCR in HMEC suspended cells, and (c) induction of MnSOD in MCF10A suspended cells at the indicated time points 
(ref. 5). Indeed, induction of MnSOD was even more robust in ERR $\gamma$-depleted cells than that in control cells (Supplementary Figure S1A). Similar induction of MnSOD was observed in PDK4-depleted cells (not shown), suggesting that induction of MnSOD by ECM detachment is not dependent on activation of the ERR $\gamma$-PDK4 axis.

Transcription of MnSOD is known to be directly activated by several transcription factors under constitutive or stress conditions. ${ }^{11}$ The forkhead domain transcription factor FOXO3a can bind to the promoter region of MnSOD and activate its expression. ${ }^{12}$ Matrix attachment activates the PI3 kinase pathway, ${ }^{1}$ which inhibits FOXO3a. Therefore, FOXO3a is likely to be activated by cell detachment. However, we observed that induction of MnSOD following detachment was not impaired in FOXO3a-depleted cells (Supplementary Figure $\mathrm{S} 1 \mathrm{~B}$ ), suggesting that $\mathrm{FOXO3a}$ is not required for activation of MnSOD in suspended cells.

The NF $\kappa \mathrm{B}$ family of transcription factors is well-established transcriptional regulators of MnSOD. ${ }^{11}$ Moreover, NF $\kappa$ B was shown to be activated following cell detachment: matrix detachment caused phosphorylation and degradation of $\mathrm{I}_{\kappa} \mathrm{B} \alpha$ (a negative regulator of $\mathrm{NF} \kappa \mathrm{B}$ ), and consequently nuclear translocation of p65 (also known as RelA) and subsequent transcriptional activation of downstream target genes. ${ }^{13} \mathrm{We}$ tested whether $\mathrm{NF}_{\kappa} \mathrm{B}$ is responsible for induction of MnSOD in detached cells. MCF10A cells were transduced with lentiviral short-hairpin RNA (shRNA) targeting p65. Quantitative RTPCR confirmed efficient knockdown of p65 in both attached and suspended cells (Figure 2a), even though unexpectedly, p65 was also upregulated when MCF10A cells were cultured in suspension (Figure 2a). We then examined the effect of p65 knockdown on induction of MnSOD by matrix detachment. In adherent cells, depletion of p65 did not influence the basal levels of MnSOD RNA (Figure 2b), implying that $N F \kappa B$ is probably not activated in MCF10A cells under attached condition. When cells were detached, induction of MnSOD in p65-depleted cells was substantially impaired compared with control cells (Figure $2 \mathrm{~b}$ ). This result suggests that $\mathrm{NF}_{\kappa} \mathrm{B}$, which is activated by cell detachment, is the primary activator that drives MnSOD expression in suspended cells.

Depletion of MnSOD sensitizes mammary epithelial cells to anoikis. It is imperative to elucidate the significance of MnSOD upregulation in response to cell detachment.
MnSOD was depleted in MCF10A cells using retroviral shRNA and the knockdown efficiency was confirmed by western blotting. Consistent with induction of MnSOD RNA (Figure 1), MnSOD protein levels increased when cells were detached (Figure 3a). The shRNA efficiently reduced MnSOD protein levels in cells under attached and detached conditions (Figure 3a).

We then investigated the effect of MnSOD depletion on anoikis. Overall cell viability was determined by Trypan Blue exclusion assay. While depletion of MnSOD exhibited little effect on cell viability in attached cells (Figure 3b), it caused significantly more cell death when cells were under suspension conditions (Figure 3b). To verify if the increased cell death was attributed to apoptosis, we measured caspase $3 / 7$ activity and performed phycoerythrin (PE) Annexin V/7-AAD staining analysis. Under attached conditions, both control cells and MnSOD-depleted cells displayed low caspase $3 / 7$ activity (Figure 3c). When detached, control cells showed strong induction of caspase activity, and depletion of MnSOD further enhanced this induction (Figure 3c). In the Annexin V analysis, compared with control cells, MnSOD-depleted cells exhibited elevated apoptosis when detached from matrix (Figures $3 d$ and e). No significant increase in apoptosis was observed in MnSOD-depleted MCF10A cells under adherent culture condition. Therefore, depletion of MnSOD enhances anoikis. Collectively, these findings suggest that induction of MnSOD in response to matrix detachment protects suspended cells from apoptosis.

Depletion of MnSOD results in accumulation of mitochondrial superoxide in detached cells. MnSOD catalyzes the conversion of the highly reactive and unstable superoxide anion in mitochondria to less reactive and more stable hydrogen peroxide, a critical step in ROS detoxification. Because depletion of MnSOD enhances anoikis, we expect it is likely due to increased accumulation of mitochondrial superoxide. To verify this idea, we measured mitochondrial superoxide levels using MitoSOX.

When MCF10A cells were detached, there was a marked increase in the amount of mitochondrial superoxide (Figures $4 \mathrm{a}$ and b). Compared with control cells, MnSOD-depleted cells slightly increased superoxide levels under attached conditions, but accumulated significantly more superoxide in mitochondria when detached (Figures $4 a$ and b). This
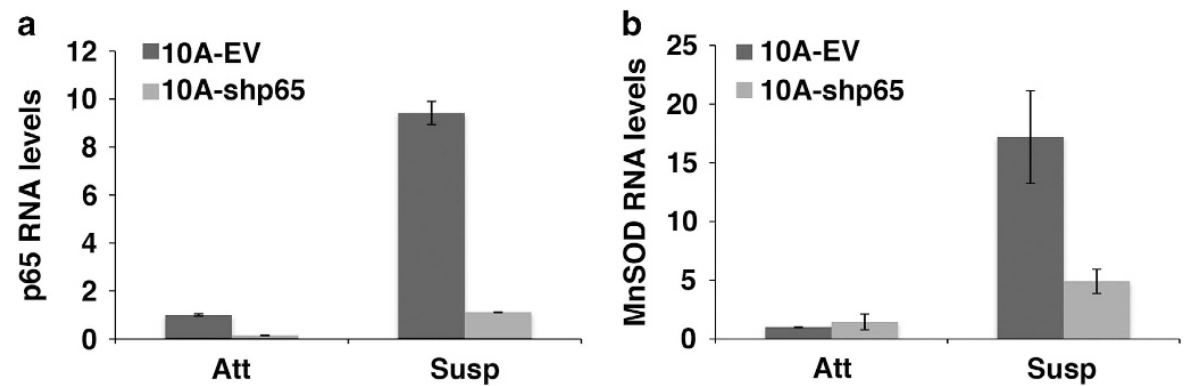

Figure 2 Depletion of $\mathrm{NF} \kappa \mathrm{B}$ undermines MnSOD induction in suspended MCF10A cells. MCF10A cells were transduced with a lentiviral empty vector (EV) or shRNA targeting p65 (shp65) and cultured under attached (Att) and suspended (Susp) conditions for $24 \mathrm{~h}$. (a) Quantitative RT-PCR measurement for p65 in MCF10A cells under attached (Att) and suspended (Susp) conditions and (b) quantitative RT-PCR measurement for MnSOD in MCF10A cells under attached (Att) and suspended (Susp) conditions. Error bars represent standard deviation 


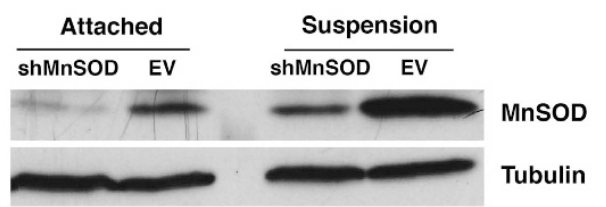

b

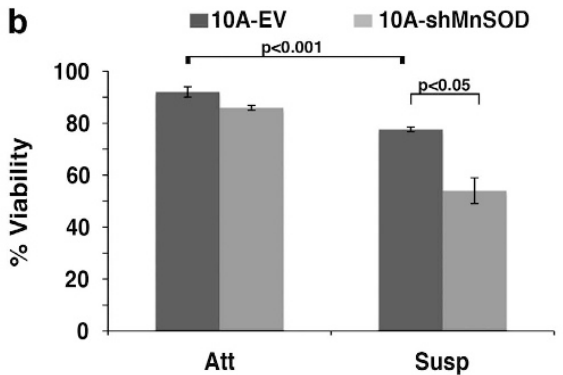

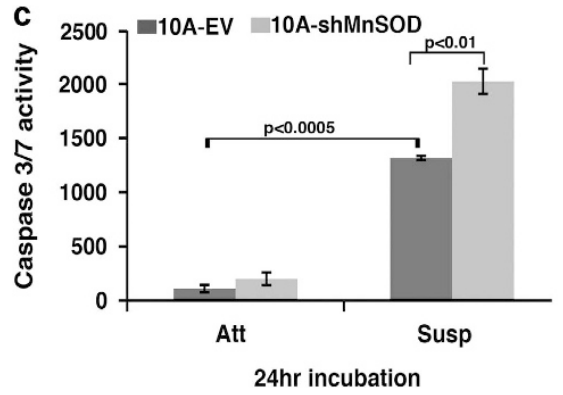
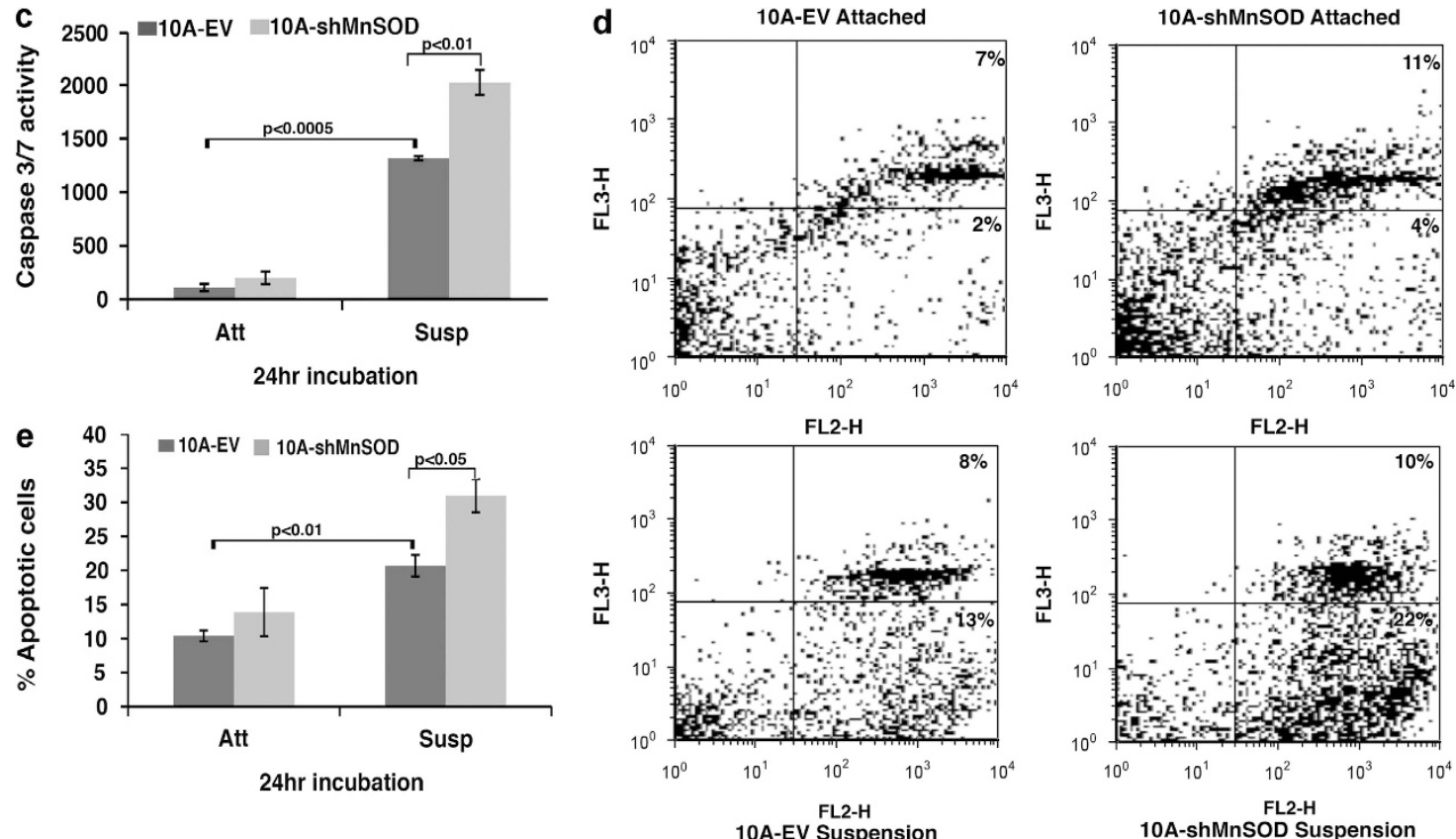

Figure 3 Depletion of MnSOD enhances anoikis in MCF10A cells. MCF10A cells were transduced with a retroviral vector (EV) or shRNA targeting MnSOD (shMnSOD) and cultured under attached (Att) and suspended (Susp) conditions for $24 \mathrm{~h}$. (a) Western blotting of MnSOD depletion in MCF10A cells under attached and suspended conditions. $\alpha$-Tubulin was used as a loading control. (b) Trypan blue exclusion assay of cell viability in control and MnSOD-depleted MCF10A cells under attached and suspended conditions. (c) Measurement of caspase 3/7 activity in control and MnSOD-depleted MCF10A cells. (d) Fluorescence-activated cell sorting analysis of Annexin V/7AAD staining in control and MnSOD-depleted MCF10A cells. The x-axes show Annexin V staining, and Y-axes show 7-AAD staining. (e) Statistics of total apoptotic cells based on the Annexin V/7-AAD analysis. Error bars represent standard deviations

observation validates that matrix detachment-induced MnSOD diminishes the level of mitochondrial superoxide generated by the detachment process.

Consistent with the essential role of $\mathrm{NF}_{\kappa} \mathrm{B}$ in activation of MnSOD following cell detachment (Figure 2), p65-depleted cells also displayed higher mitochondrial superoxide levels than control cells under suspension culture conditions (Figures $4 \mathrm{a}$ and $\mathrm{b}$ ). Together, these findings suggest that in response to matrix detachment, cells activate the $\mathrm{NF} \kappa \mathrm{B}$ MnSOD axis to detoxify mitochondrial ROS that are induced by detachment (Figure 5a). Depletion of MnSOD or $\mathrm{NF}_{\kappa} \mathrm{B}$ increases the toxic superoxide free radical in suspended cells, thereby sensitizing cells to anoikis.

MnSOD depletion and PDK inhibition collaboratively enhance anoikis. In addition to activation of $\mathrm{NF}_{\kappa} \mathrm{B}-\mathrm{MnSOD}$ upon cell detachment, we recently observed that detached mammary epithelial cells upregulate the ERR $\gamma$-PDK4 pathway to attenuate PDH activity and oxidative metabolism of glycolysis-derived pyruvate. ${ }^{5}$ This metabolic response enables suspended cells to avoid excessive ROS generation and acquire resistance to anoikis. ${ }^{5}$ Therefore, detached cells manage to contain ROS levels by reducing mitochondrial ROS production and concomitantly increasing antioxidant capacity to detoxify ROS (Figure 5a). These responses endow cells with prolonged viability in suspension.

We asked whether simultaneous interruption of these two pathways may lead to a synergistic effect on anoikis. Dichloroacetate (DCA) is a weak inhibitor of PDKs, and can modestly shift pyruvate metabolism from lactate to acetyl-CoA by activation of $\mathrm{PDH} .{ }^{14,15}$ This action leads to increased oxygen consumption and accompanied generation of ROS. DCA treatment alone had limited influence on anoikis in MCF10A cells, however, it strongly stimulated anoikis in MnSOD-depleted cells (Figure 5b). Therefore, these results suggest that increased oxidative metabolism of glucose and decreased antioxidant capacity cooperatively accelerate anoikis in mammary epithelial cells. 
a Attached

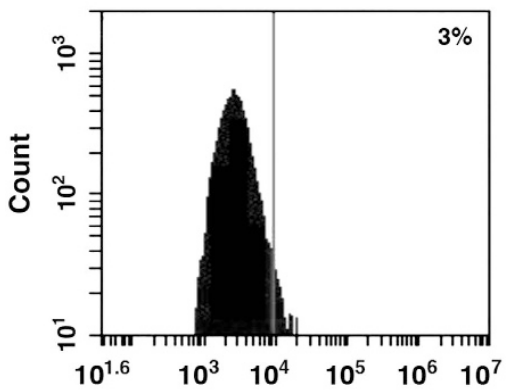

Suspension
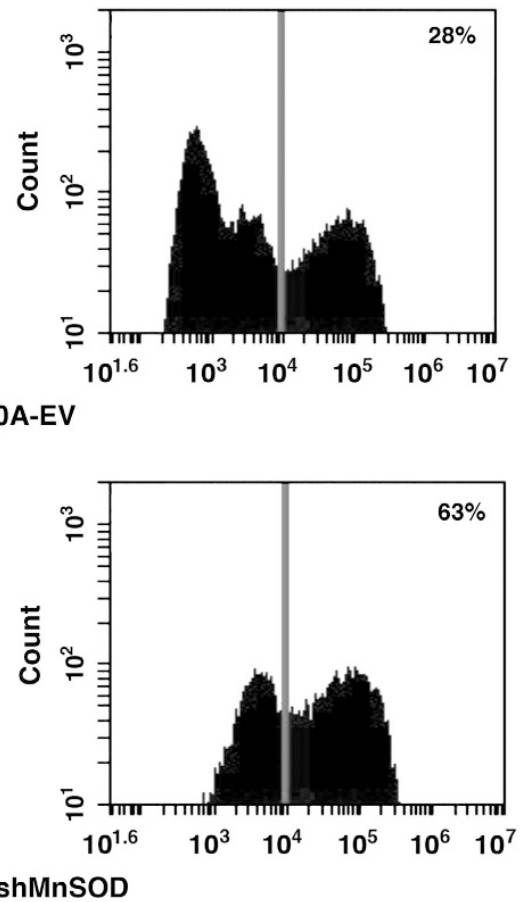

10A-shMnSOD
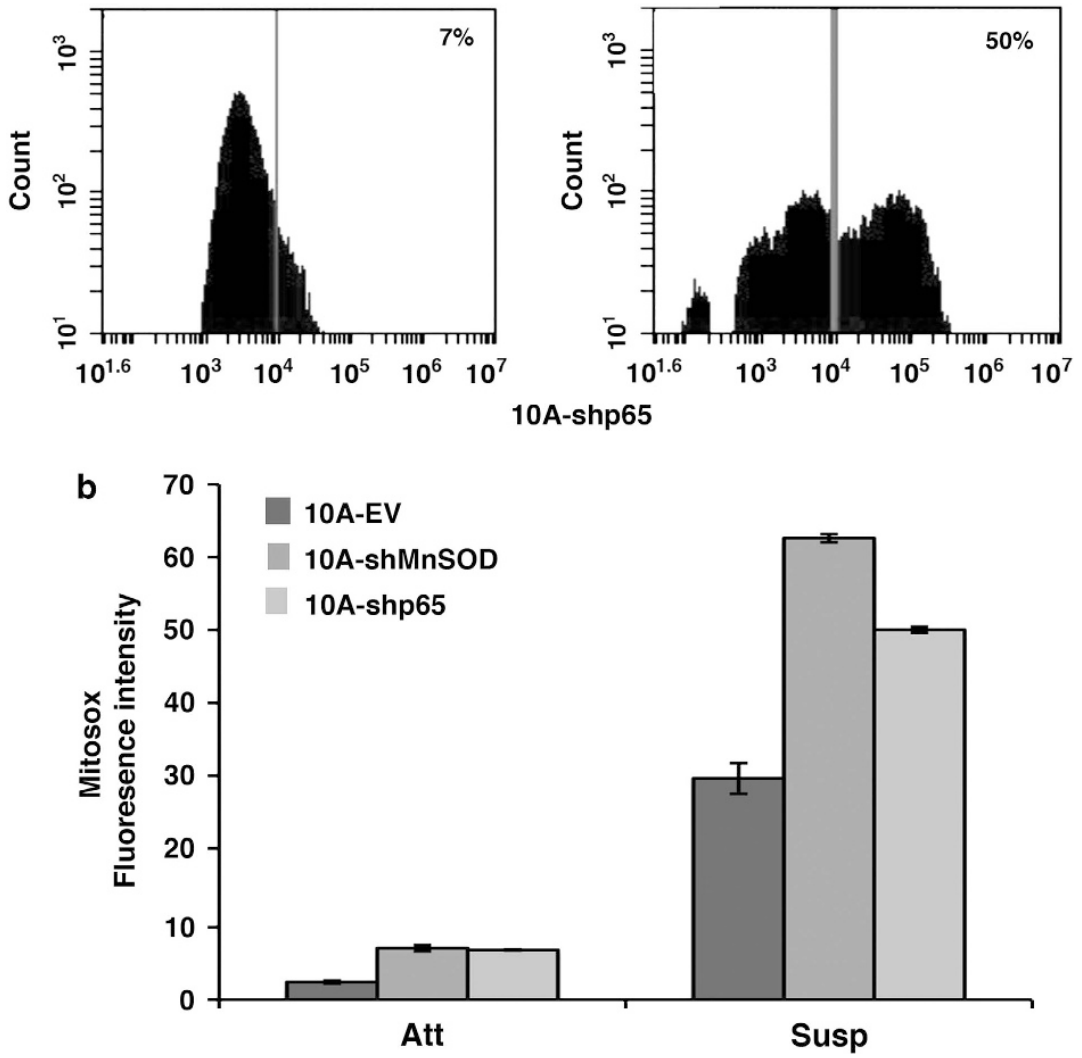

Figure 4 Depletion of MnSOD or p65 escalates accumulation of mitochondrial superoxide in suspended MCF10A cells. Empty vector (EV), shRNA targeting MnSOD (shMnSOD), and shRNA targeting p65 (shp65) are cultured under attached (Att) or suspended (Susp) conditions for $24 \mathrm{~h}$, incubated with $5 \mu \mathrm{M}$ Mitosox red for 30 min at $37^{\circ} \mathrm{C}$ incubator, trypsinized, washed with PBS, and ran samples on flow cytometry with $488 \mathrm{~nm}$ excitation to measure oxidized Mitosox red in the FL2 and FL3 channels. Collected 10,000 events per sample. (a) Mitosox red measurement in EV, shMnSOD, and shp65, and (b) statistics of mean intensity of Mitosox red based on the analysis 
a

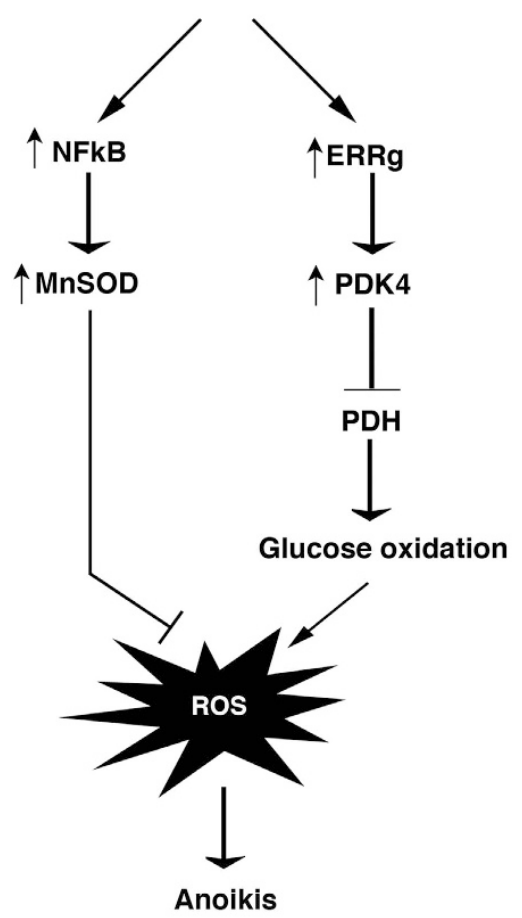

b
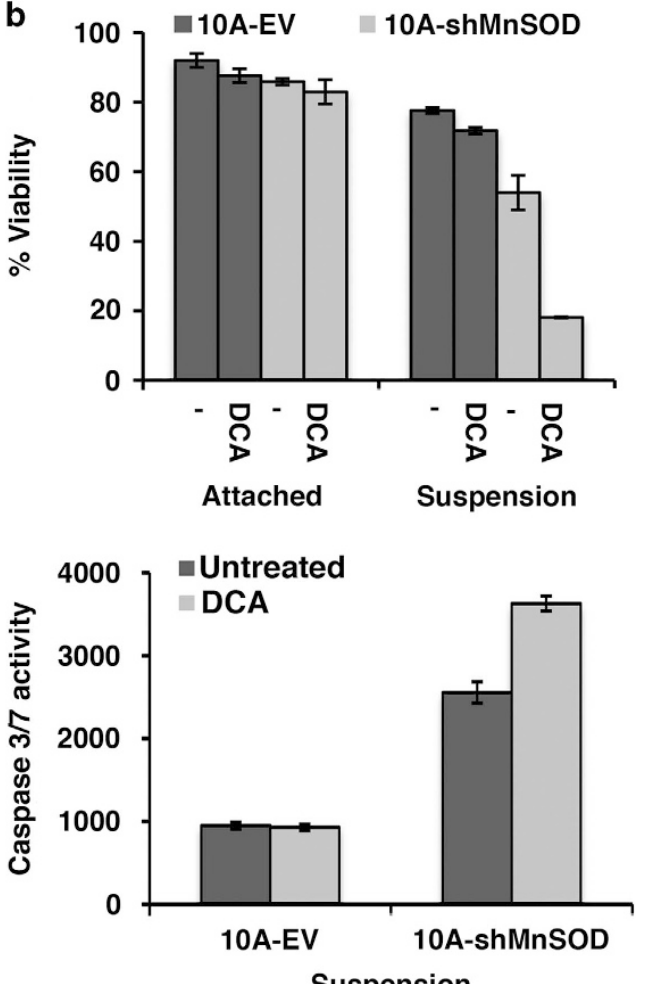

Figure 5 Matrix detachment activates $\mathrm{NF}_{\kappa} \mathrm{B}$ - and $\mathrm{ERR} \gamma$-dependent transcription programs to induce MnSOD and inhibit mitochondrial oxidative metabolism of glucose, respectively. (a) Schematic of metabolic reprogramming by activation of ERR $\gamma$-PDK4 and antioxidant modulation by activation of the NF $\kappa$ B-MnSOD pathway in untransformed mammary epithelial cells following matrix detachment. (b) Depletion of MnSOD and inhibition of PDKs collectively enhances anoikis in MCF10A cells. MCF10A cells transduced with empty vector (EV) or shRNA targeting MnSOD (shMnSOD) are cultured with or without $5 \mathrm{mM} \mathrm{DCA}$ under attached and suspended conditions for $24 \mathrm{~h}$ followed by Trypan blue exclusion assay of cell viability and measurement of caspase $3 / 7$ activity

MnSOD in human cancer progression. Resistance to anoikis is a prerequisite for metastasis. Given MnSOD's anti-anoikis activity, we examined its expression in human breast cancer samples by immunohistochemistry to investigate its potential implication in tumor progression. We observed that isolated, migrating cancer cells at the invasive front of primary tumor exhibited higher MnSOD expression than those inside the tumor mass (Figure 6a). We then compared MnSOD levels in primary tumors and matched lymph-node metastases. Both micrometastasis and established macrometastatic tumor cells showed elevated levels of MnSOD as compared with their corresponding primary tumors (Figures $6 \mathrm{~b}$ and $\mathrm{c}$, respectively). Moreover, metastases displayed higher percentage of MnSOD positivity than primary breast tumors (Supplementary Figure S2).

We further analyzed microarray gene expression data sets that typically contain much more cancer samples. The GSE3494 data set included primary breast tumor samples from a cohort of 315 women with clinico-pathological characteristics such as tumor grade, p53 mutation and nuclear hormone receptor status. ${ }^{16}$ We found that high MnSOD expression evidently correlated with advanced tumor grade, and overlapped with other poor prognostic markers such as p53 mutation as well as negative status of estrogen receptor $(\mathrm{ER})$ and progesterone receptor (PgR) (Figure 7a).
To determine whether MnSOD might have prognostic value, we compared MnSOD expression and patient survival by univariate analysis. Patients grouped into the high-expression tertile had significantly shorter disease-specific survival than the low-expression group (Figure $7 \mathrm{~b}$ ). These results suggest that $\mathrm{MnSOD}$ is associated with tumor progression and is a predictor of poor prognosis.

To confirm that MnSOD contributes to anoikis resistance in cancer cells, we depleted MnSOD in MDA-MB-435 cancer cells which express relatively high levels of MnSOD. Depletion of MnSOD had no notable effect on cells under attached condition. Unlike control cells whose viability was unaffected by matrix detachment, MnSOD-depleted cancer cells exhibited increased cell death when detached (Figure 7c), suggesting that cancer cell's resistance to anoikis is in part attributable to MnSOD. DCA treatment alone also restored some anoikis in cancer cells, and caused more death of detached cells when combined with depletion of MnSOD (Figure 7c).

\section{Discussion}

Normal cells rely on adhesion to appropriate ECM for survival and loss of this adhesion induces anoikis. ${ }^{1}$ Matrix detachment increases oxidative stress that has a critical role in anoikis induction. ${ }^{3}$ In the present study, we show that upon cell 

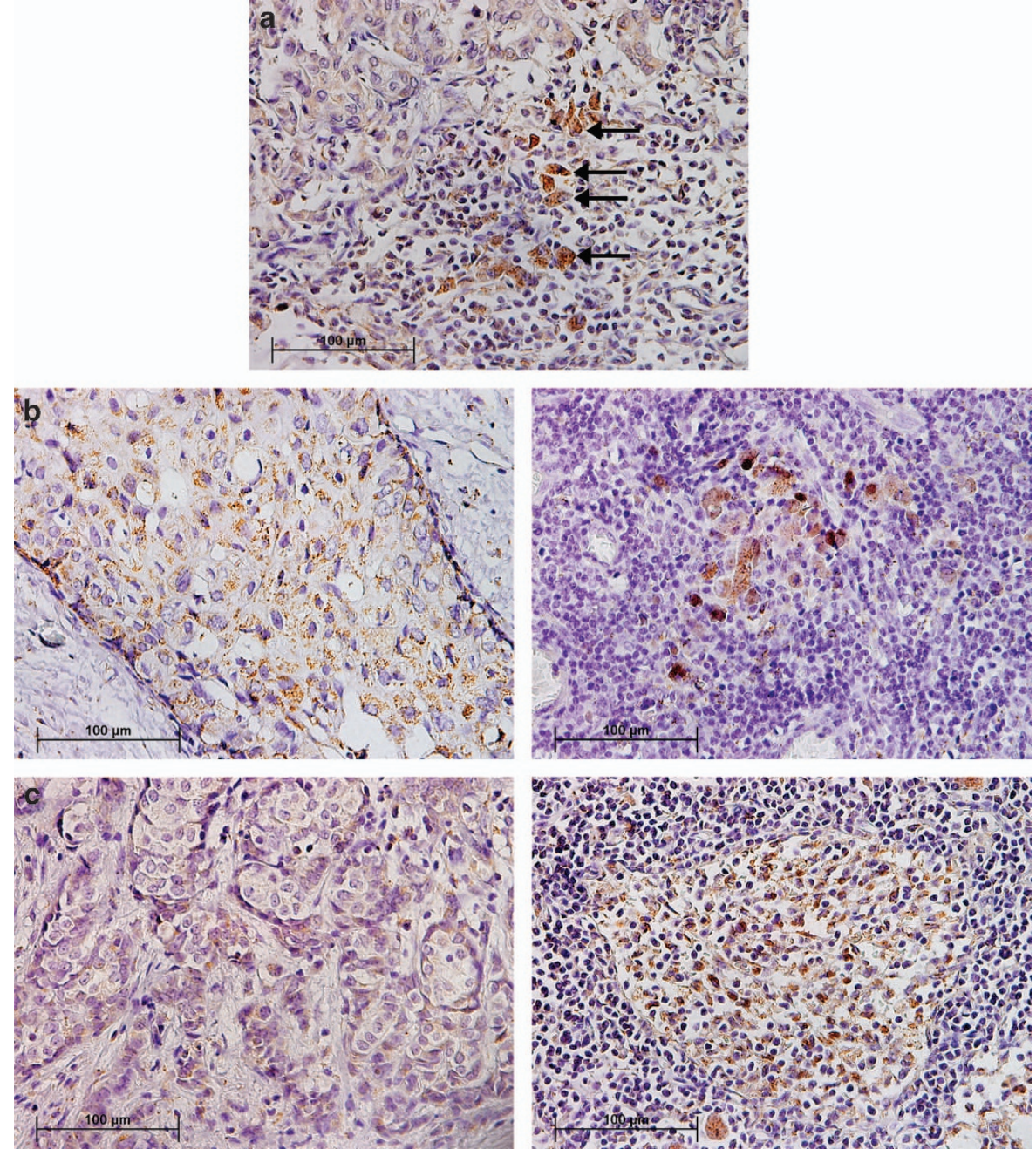

Figure 6 Expression of MnSOD in human breast cancer. (a) Migrating cancer cells (indicated by arrows) show stronger MnSOD staining than tumor cells in primary tumor mass (upper left corner). (b) A small cluster of tumor cells (micrometastasis) in lymph node (right) exhibits higher MnSOD expression than cells in matched primary tumor (left). (c) Tumor cells in established lymph-node macrometastasis (right) display increased MnSOD expression compared with those in matched primary tumor (left)

detachment, untransformed human mammary epithelial cells upregulate MnSOD, a key enzyme to detoxify mitochondrial ROS. Induction of MnSOD by detachment allows cells to remove mitochondrial superoxide and survive longer in suspension. Depletion of MnSOD sensitizes cells to anoikis. Therefore, MnSOD acts as a negative modulator of anoikis. ROS are normally produced in mitochondria during aerobic metabolism due to ineffective electron transport. We recently discovered that detached cells attenuate oxidative metabolism of glucose to reduce the associated generation of ROS. $^{5}$ Taken together, in response to matrix detachment, cells activate at least two parallel programs - increasing ROS-scavenging capacity and decreasing mitochondrial oxidative metabolism - to counter cellular oxidative stress generated by detachment and thus delay anoikis (Figure 5a).

We have found that upregulation of MnSOD in detached cells depends on $\mathrm{NF} \kappa \mathrm{B}$, which is activated by matrix detachment, ${ }^{13}$ although the underlying mechanism is not fully understood. $\mathrm{NF} \kappa \mathrm{B}$ is known to be activated by $\mathrm{ROS}^{17}$
Because matrix detachment robustly increases mitochondrial ROS levels in endothelial cells ${ }^{3}$ and in mammary epithelial cells (Figure 4), a likely scenario is that matrix detachment increases cellular ROS levels, leading to activation of the $\mathrm{NF}_{\kappa} \mathrm{B}$ pathway, which in turn induces MnSOD expression to scavenge ROS. Therefore, activation of $\mathrm{NF} \kappa \mathrm{B}-\mathrm{MnSOD}$ functions as an inducible, negative feedback mechanism to control ROS levels in suspended cells. NF $\kappa \mathrm{B}$ has wellestablished pro-survival activity. Inhibition of $\mathrm{NF}_{\kappa} \mathrm{B}$ results in accelerated apoptosis in suspended cells. ${ }^{13}$ Similarly, depletion of MnSOD enhances anoikis (Figure 3), suggesting that MnSOD may be an essential mediator of NF $\kappa$ B's pro-survival activity in suspended cells.

Anoikis resistance is essential for tumor metastasis. ${ }^{18}$ Resistance to anoikis enables adherent cells to survive under suspension condition and at ectopic sites where the matrix components are different from those the cells originally contact. The anti-anoikis activity implicates MnSOD in tumor metastatic progression. While expression of MnSOD may vary during cancer development (depending on the type and 

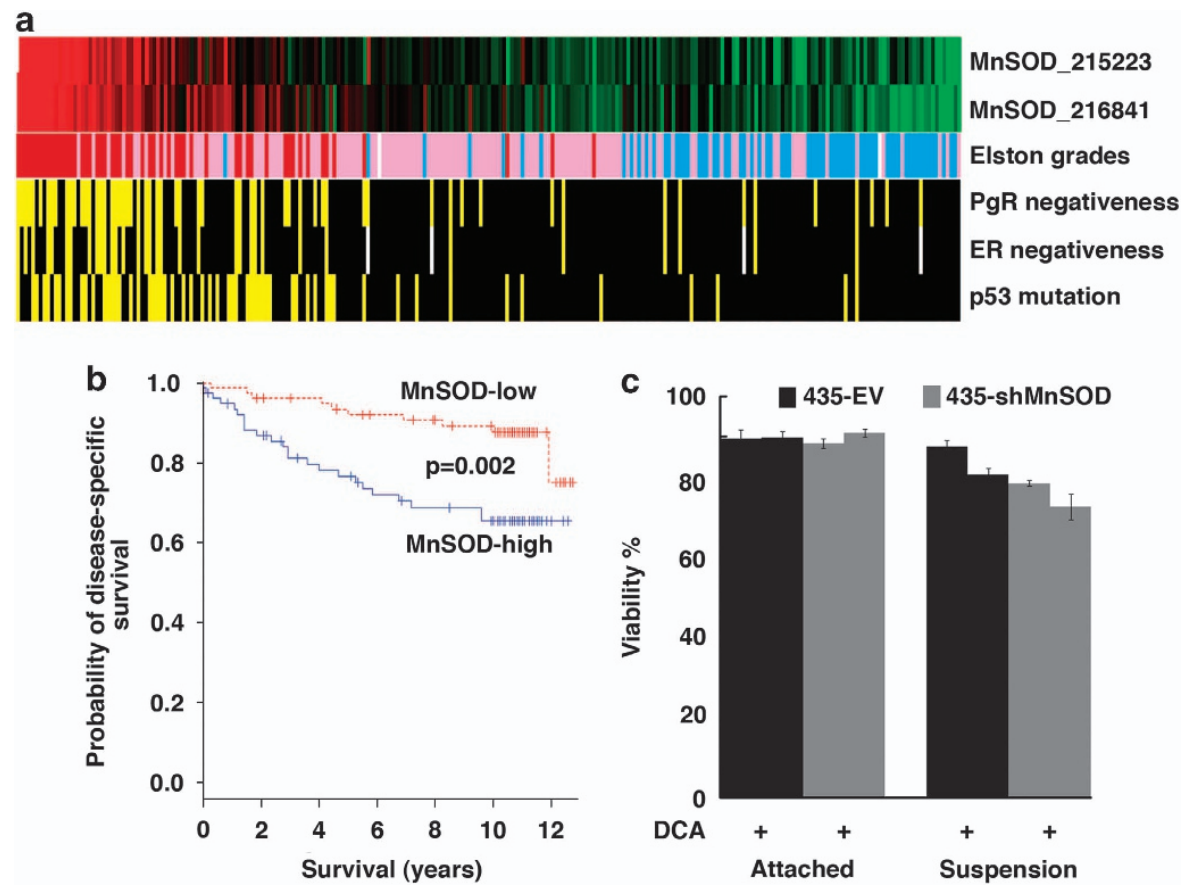

Figure 7 Expression and function of MnSOD in human cancer. (a) Elevated expression of MnSOD (high: red; low: green) is associated with high Elston-Ellis grades of the tumors (high: red; medium: pink; low: blue), negative status of progesterone receptor (PgR) and estrogen receptor (ER) (negative: yellow), and p53 mutation (mutation: yellow). The microarray data GSE3494 was downloaded from the Gene Expression Omnibus. MnSOD RNA levels were detected by two indicated cDNA probes. (b) MnSOD inversely correlates with 10-year patient survival. (c) Depletion of MnSOD in MDA-MB-435 human cancer cells restores their sensitivity to matrix detachment

stage), accumulating evidence shows that MnSOD levels in human cancer often correlate with the degree of malignancy. ${ }^{19}$ In our analysis of human breast cancer, elevated expression of MnSOD is observed in invading and metastatic tumor cells (Figure 6) as compared with matched primary tumors, and is significantly associated with advanced histological grades, negative status of hormone receptors, mutated p53, as well as poor prognosis (Figures 7a and b). The prosurvival effect of MnSOD may also promote resistance to chemo- and radiation-therapies that exert cytotoxic effects in part by inducing oxidative damage. ${ }^{20,21}$

Cancerous tissues were reported to produce increased amounts of ROS. ${ }^{22}$ Low levels of ROS activate signaling molecules/pathways (including Src, PI3K, NF $\kappa$ B, and HIF) that may increase cell proliferation and survival under mild oxidative conditions, and contribute to cellular transformation. ${ }^{23}$ By contrast, high ROS content above that required for signaling may cause overwhelming oxidative damage that can culminate in senescence or death in both normal and cancer cells. Indeed, common cancer therapies may kill cancer cells by promoting oxidative stress. ${ }^{9}$ To cope with high oxidative stress, cancer cells develop enhanced antioxidant systems. ${ }^{9,24}$ The pro-malignant potential of antioxidants has been increasingly recognized. Besides MnSOD, other important cellular antioxidants (e.g., thioredoxin, glutaredoxin, and peroxiredoxin) are upregulated in some cancer cells. ${ }^{25}$ The transcription factor Nrf2-dependent antioxidant program is often activated in human cancer due to somatic mutations and is essential for tumorigenesis in vivo. ${ }^{26,27}$ Indeed, we found that like MnSOD, NRF2 also confers anoikis resistance
(Supplementary Figure S3). Consistent with these observations, when used as dietary supplements, antioxidants appear to have an adverse effect on the cancer incidence ${ }^{28}$ and protect tumor cells against therapy. ${ }^{29}$

In addition to enhanced antioxidant capacity, most cancer cells exhibit altered glucose metabolism with a preference for aerobic glycolysis (known as the Warburg effect). ${ }^{30}$ Many malignant cells metabolize glucose via pyruvate preferentially to lactate instead to $\mathrm{CO}_{2}$ and $\mathrm{H}_{2} \mathrm{O}$ through mitochondrial oxidative metabolism even in the presence of oxygen. We recently showed that the Warburg metabolic phenotype allows cancer cells to evade excessive ROS generation associated with mitochondrial respiration and acquire a survival advantage when detached, thereby contributing to anoikis resistance. ${ }^{5}$ In the wake of these findings, a combination treatment to pharmacologically target cell's capacity of ROS detoxification ${ }^{9,31}$ and normalize glucose metabolism (e.g., activation of PDH) may maximally provoke oxidative stress in cancer cells, which may represent an effective strategy to lower the threshold for apoptosis, restore anoikis sensitivity, and reduce metastasis and therapy resistance.

\section{Materials and Methods}

Cells and reagents. MCF10A (human mammary epithelial cell line) and HMECs were cultured in Dulbecco' modified Eagle medium (DMEM)/F-12 medium (Cellgro, Manassas, VA, USA) supplemented with 5\% horse serum (Sigma, St Louis, MO, USA), $20 \mathrm{ng} / \mathrm{ml}$ epidermal growth factor (EGF; Sigma), $10 \mu \mathrm{g} / \mathrm{ml}$ insulin (Sigma), and $0.5 \mu \mathrm{g} / \mathrm{ml}$ hydrocortisone (Sigma). All assays in untransformed cells and HMECs were performed $24 \mathrm{~h}$ after incubation under attached and suspended conditions unless otherwise noted. DCA was purchased from Sigma. 
RNA isolation and quantitative RT-PCR. Total RNA isolation from cells grown under attached and suspended conditions was carried out with TRIzol (Invitrogen, Grand Island, NY, USA), following manufacturer's protocol. For quantitative reverse transcription-polymerase chain reaction (RT-PCR), cDNA was synthesized using Moloney murine leukemia virus (M-MuLV) reverse transcriptase with random primers. Real-time PCR was performed with SYBR green PCR mix (Applied Biosystems, Foster City, CA, USA). The PCR cycling conditions were as follows: 40 cycles of $30 \mathrm{~s}$ at $95^{\circ} \mathrm{C}, 30 \mathrm{~s}$ at $60^{\circ} \mathrm{C}$, and $30 \mathrm{~s}$ at $72^{\circ} \mathrm{C}$. $\beta$-Actin was used for normalization. Error bars represent standard deviations $(n=3)$. PDK expression under attached conditions was set as 1 .

The primers used for the MnSOD, p65, ERR $\gamma$, FOXO3a, and $\beta$-actin were the following MnSOD, forward, $5^{\prime}$-TACGTGAACAACCTGAAC- $3^{\prime}$, and reverse, $5^{\prime}$-TAT CTGGGCTGTAACATCT-3'; ERR $\gamma$, forward, 5'-TCTTGCTAATTCAGACTCCAT-3', and reverse, $5^{\prime}$-GCAGTGTCATCAGCATCTTG-3'; FOXO3a, forward, 5'-CTGAAC TCCCTACGCCAGTC- $3^{\prime}$, and reverse, $5^{\prime}$-GAGTCCGAAGTGAGCAGGTC- ${ }^{\prime}$ (ref. 32); $\beta$-actin, forward, 5'-AGAAAATCTGGCACCACACC-3', and reverse, 5'-AGAGG CGTACAGGGATAGCA-3'.

Depletion of MnSOD, p65, ERR $\gamma$, FOXO3a, and NRF2 by retroviral and lentiviral shRNA transduction. MnSOD gene was depleted in MCF10A by retroviral transduction using the MSCV-LMP vector (OpenBiosystems, Waltham, MA, USA). The shRNA targeting sequence for MnSOD was 22 mer $5^{\prime}$-AAGGAACAACAGGCCTTATTCC-3' (ref. 33). Lentiviral shRNAs targeting ERR $\gamma$, FOXO3a, NRF2, and p65 were obtained from OpenBiosystems. The ERR $\gamma$ shRNA target sequence was $5^{\prime}$-CAGTGGGAGCTACAGTT CA-3' (ref. 34).

Trypan blue exclusion assay and caspase $3 / 7$ activity assay. Cell viability was determined using $0.4 \%$ Trypan blue dye in a hemacytometer. Dead cells were stained blue. The caspase $3 / 7$ assay was performed using Caspase 3/7 Glo assay kit from Promega, Madison, WI, USA. Cells were lysed in hypertonic buffer (HTB; $10 \mathrm{mM}$ HEPES (pH 7.9), $1.5 \mathrm{mM} \mathrm{MgCl}_{2}, 10 \mathrm{mM} \mathrm{KCl}$, adding $0.2 \mathrm{mM}$ phenylmethylsulfonyl fluoride (PMSF); $0.5 \mathrm{M}$ DTT freshly added before use). The protein concentration of samples was measured using the BCA kit (Pierce, Rockford, IL, USA). Aliquots of $75-100 \mu \mathrm{g}$ of protein was loaded in each of the 96-well microplates, and the caspase 3/7 assay was performed following manufacturer's instructions.

Annexin V/7-AAD analysis. Annexin V/7-AAD was performed as previously described. ${ }^{5} \mathrm{~A}$ total of $1 \times 10^{5}$ cells were washed twice with PBS, followed by staining with PE-Annexin $V$ and $7-A A D$ for $15 \mathrm{~min}$ at room temperature, following manufacturer's instruction. Cells were analyzed within an hour using Becton Dickson's Facsort apparatus (Franklin Lakes, NJ, USA). CellQuest software (BD Biosciences, San Jose, CA, USA) was used to analyze the data as an [FL2H, FL3H] log-scale two-dimensional diagram.

MitoSOX Red analysis. Cells were grown under attached and suspended culture conditions. After $24 \mathrm{~h}$ of incubation, the cells were incubated with $5 \mu \mathrm{M}$ Mitosox Red dye for $30 \mathrm{~min}$ at $37^{\circ} \mathrm{C}$ incubator avoiding light exposure. Later, cells were trypsinized, washed with PBS and resuspended at $1 \times 10^{7}$ cells per $\mathrm{ml}$. The cells were placed in FACS tube at a concentration of $5 \times 10^{6}$ cells per $100 \mu \mathrm{l}$ and diluted to a final volume of $500 \mu \mathrm{l}$ with FACS binding buffer. Using $488 \mathrm{~nm}$ excitation, the samples were run on flow cytometry to measure oxidized Mitosox Red in the $\mathrm{FL}_{2}$ and $\mathrm{FL}_{3}$ channels. Each sample was collected using 10000 events.

Immunohistochemistry. Formalin-fixed paraffin-embedded human breast cancer sections were deparaffinized, rehydrated, and stained with primary antibodies (1:500, anti-MnSOD2 sc-30080; Santa Cruz, Dallas, TX, USA). After incubating with the specific HRP-conjugated secondary antibody, DAB was applied to the tissue. Sections were counterstained with hematoxylin. Positively stained cells were counted in three high power field areas ( $\times 100$ oil immersion) in each breast section. No staining was observed in tissues incubated with PBS as negative control. Paired $T$-test was employed for statistics.

\section{Conflict of Interest}

The authors declare no conflict of interest.
Acknowledgements. This work was supported by grants from the National Cancer Institute (R01CA137021) and Florida Bankhead-Coley Cancer Research Program (09BN-12-23092 and 2BT01).

1. Gilmore AP. Anoikis. Cell Death Differ. 2005; 12: 1473-1477.

2. Llambi F, Moldoveanu T, Tait SW, Bouchier-Hayes L, Temirov J, McCormick LL et al. A unified model of mammalian BCL-2 protein family interactions at the mitochondria. Mol Cell 2011; 44: 517-531.

3. Li AE, Ito H, Rovira II, Kim KS, Takeda K, Yu ZY et al. A role for reactive oxygen species in endothelial cell anoikis. Circ Res 1999; 85: 304-310.

4. Orrenius S, Gogvadze V, Zhivotovsky B. Mitochondrial oxidative stress: implications for cell death. Annu Rev Pharmacol Toxicol 2007; 47: 143-183.

5. Kamarajugadda S, Stemboroski L, Cai Q, Simpson NE, Nayak S, Tan M et al. Glucose oxidation modulates anoikis and tumor metastasis. Mol Cell Biol 2012; 32: 1893-1907.

6. Schafer ZT, Grassian AR, Song L, Jiang Z, Gerhart-Hines Z, Irie HY et al. Antioxidant and oncogene rescue of metabolic defects caused by loss of matrix attachment. Nature 2009; 461: 109-113.

7. Giannoni E, Buricchi F, Grimaldi G, Parri M, Cialdai F, Taddei ML et al. Redox regulation of anoikis: reactive oxygen species as essential mediators of cell survival. Cell Death Differ 2008; 15: 867-878.

8. Stowe DF, Camara AK. Mitochondrial reactive oxygen species production in excitable cells: modulators of mitochondrial and cell function. Antioxid Redox Signal 2009; 11: 1373-1414

9. Trachootham D, Alexandre J, Huang P. Targeting cancer cells by ROS-mediated mechanisms: a radical therapeutic approach? Nat Rev Drug Discov 2009; 8: 579-591.

10. Grassian AR, Metallo CM, Coloff JL, Stephanopoulos G, Brugge JS. Erk regulation of pyruvate dehydrogenase flux through PDK4 modulates cell proliferation. Genes Dev 2011; 25: $1716-1733$.

11. Dhar SK, St Clair DK. Manganese superoxide dismutase regulation and cancer. Free Radic Biol Med 2012; 52: 2209-2222.

12. Kops GJ, Dansen TB, Polderman PE, Saarloos I, Wirtz KW, Coffer PJ et al. Forkhead transcription factor $\mathrm{FOXO} 3$ a protects quiescent cells from oxidative stress. Nature 2002; 419: 316-321

13. Yan SR, Joseph RR, Rosen K, Reginato MJ, Jackson A, Allaire $\mathrm{N}$ et al. Activation of NF-kappaB following detachment delays apoptosis in intestinal epithelial cells. Oncogene 2005; 24: 6482-6491.

14. Stacpoole PW, Kurtz TL, Han Z, Langaee T. Role of dichloroacetate in the treatment of genetic mitochondrial diseases. Adv Drug Deliv Rev 2008; 60: 1478-1487.

15. Michelakis ED, Webster L, Mackey JR. Dichloroacetate (DCA) as a potential metabolictargeting therapy for cancer. Br J Cancer 2008; 99: 989-994.

16. Miller LD, Smeds J, George J, Vega VB, Vergara L, Ploner A et al. An expression signature for p53 status in human breast cancer predicts mutation status, transcriptional effects, and patient survival. Proc Natl Acad Sci USA 2005; 102: 13550-13555.

17. Morgan MJ, Liu ZG. Crosstalk of reactive oxygen species and NF- $\mathrm{KB}$ signaling. Cell Res 2011; 21: 103-115.

18. Douma S, Van Laar T, Zevenhoven J, Meuwissen R, Van Garderen E, Peeper DS. Suppression of anoikis and induction of metastasis by the neurotrophic receptor TrkB. Nature 2004; 430: 1034-1039.

19. Pani G, Colavitti R, Bedogni B, Fusco S, Ferraro D, Borrello S et al. Mitochondrial superoxide dismutase: a promising target for new anticancer therapies. Curr Med Chem 2004: 11: 1299-1308.

20. Fisher CJ, Goswami PC. Mitochondria-targeted antioxidant enzyme activity regulates radioresistance in human pancreatic cancer cells. Cancer Biol Ther 2008; 7: $1271-1279$

21. Yeung BH, Wong KY, Lin MC, Wong CK, Mashima T, Tsuruo T et al. Chemosensitisation by manganese superoxide dismutase inhibition is caspase- 9 dependent and involves extracellular signal-regulated kinase 1/2. Br J Cancer 2008; 99: 283-293.

22. Haklar G, Sayin-Ozveri E, Yüksel M, Aktan AO, Yalçin AS. Different kinds of reactive oxygen and nitrogen species were detected in colon and breast tumors. Cancer Lett 2001; 165: 219-224.

23. Gupta SC, Hevia D, Patchva S, Park B, Koh W, Aggarwal BB. Upsides and downsides of reactive oxygen species for cancer: the roles of reactive oxygen species in tumorigenesis, prevention, and therapy. Antioxid Redox Signal 2012; 16: 1295-1322.

24. Cairns RA, Harris IS, Mak TW. Regulation of cancer cell metabolism. Nat Rev Cancer 2011; 11: 85-95

25. Landriscina $M$, Maddalena $F$, Laudiero $G$, Esposito $F$. Adaptation to oxidative stress, chemoresistance, and cell survival. Antioxid Redox Signal 2009; 11: 2701-2706.

26. Hayes JD, McMahon M. NRF2 and KEAP1 mutations: permanent activation of an adaptive response in cancer. Trends Biochem Sci 2009; 34: 176-188.

27. DeNicola GM, Karreth FA, Humpton TJ, Gopinathan A, Wei C, Frese K et al. Oncogeneinduced Nrf2 transcription promotes ROS detoxification and tumorigenesis. Nature 2011; 475: 106-109.

28. Omenn GS, Goodman GE, Thornquist MD, Balmes J, Cullen MR, Glass A et al. Effects of a combination of beta carotene and vitamin $A$ on lung cancer and cardiovascular disease. N Engl J Med 1996; 334: 1150-1155. 
34. Cai Q, Lin T, Kamarajugadda S, Lu J. Regulation of glycolysis and the Warburg effect by estrogen-related receptors. Oncogene 2012; e-pub ahead of print 4 June 2012; doi:10.1038/onc.2012.221. therapy? J Natl Cancer Inst 2008; 100: 773-783.

30. Vander Heiden MG, Cantley LC, Thompson CB. Understanding the Warburg effect: the metabolic requirements of cell proliferation. Science 2009; 324: 1029-1033.

31. Montero AJ, Jassem J. Cellular redox pathways as a therapeutic target in the treatment of cancer. Drugs 2011; 71: 1385-1396.

32. Mandinova A, Lefort K, Tommasi di Vignano A, Stonely W, Ostano P, Chiorino G et al. The FoxO3a gene is a key negative target of canonical Notch signalling in the keratinocyte UVB response. EMBO J 2008; 27: 1243-1254.

33. Chen Y, Azad MB, Gibson SB. Superoxide is the major reactive oxygen species regulating autophagy. Cell Death Differ 2009; 16: 1040-1052.
Cell Death and Disease is an open-access journal published by Nature Publishing Group. This work is licensed under the Creative Commons Attribution-Non
Commercial-No Derivative Works 3.0 Unported License. To view a copy of this license, visit http://creativecommons.org/licenses/by-nc$\mathrm{nd} / 3.0 /$

Supplementary Information accompanies the paper on Cell Death and Disease website (http://www.nature.com/cddis) 